\title{
Resonant breakup of ${ }^{19} \mathrm{C}$ on a proton target
}

\author{
R. Crespo, ${ }^{1,2}$ M. Rodríguez-Gallardo,,${ }^{1,3,4}$ A. M. Moro,${ }^{4}$ A. Deltuva,,${ }^{1}$ E. Cravo,${ }^{1}$ and A. C. Fonseca ${ }^{1}$ \\ ${ }^{1}$ Centro de Física Nuclear, Universidade de Lisboa, Av. Prof. Gama Pinto 2, P-1649-003 Lisboa, Portugal \\ ${ }^{2}$ Departamento de Física, Instituto Superior Técnico, Taguspark, Av. Prof. Cavaco Silva, Taguspark, \\ P-2780-990 Porto Salvo, Oeiras, Portugal \\ ${ }^{3}$ Instituto de Estructura de la Materia, CSIC, Serrano 123, E-28006 Madrid, Spain \\ ${ }^{4}$ Departamento de Física Atómica, Molecular y Nuclear, Universidad de Sevilla, Apartado 1065, E-41080 Sevilla, Spain
}

(Received 7 February 2011; published 23 May 2011)

\begin{abstract}
The resonant breakup of ${ }^{19} \mathrm{C}$ on a proton target at $70 \mathrm{MeV} /$ nucleon is analyzed using Faddeev-Alt, Grassberger, Sandhas (Faddeev-AGS) and continuum-discretized coupled-channels (CDCC) reaction frameworks, where in both cases a three-body model $\left({ }^{18} \mathrm{C}+n+p\right)$ for the reaction is considered. Taking a ${ }^{18} \mathrm{C}+p$ potential from a global nucleon-nucleus parametrization and a $L$-independent Gaussian proton-neutron potential, both methods provide very similar results for the calculated observables. However, when this simplified proton-neutron potential is replaced by the more realistic CD-Bonn potential, the breakup cross section, calculated with the Faddeev AGS formalism, decreases by almost one order of magnitude, largely underestimating the experimental data. From this calculation, we conclude that, within a core + valence neutron model, the single-particle mechanism gives a negligible contribution to the calculated resonant breakup and therefore core-excitation mechanisms should be taken into account.
\end{abstract}

DOI: 10.1103/PhysRevC.83.054613

PACS number(s): 25.60.Gc, 21.45.-v, 24.10.Eq, 24.50.+g

\section{INTRODUCTION}

Inelastic scattering is a useful tool to study excitation modes in halo nuclei if it is possible to single out particular multipole excitations.

Recently, the resonant breakup scattering of ${ }^{19} \mathrm{C}$ on a proton target at $70 \mathrm{MeV} /$ nucleon was measured, employing the invariant mass method in inverse kinematics in which the valence neutron and the remaining ${ }^{18} \mathrm{C}$ fragment were detected in coincidence [1], thus permitting the reconstruction of the relative energy spectrum between these two fragments. This spectrum shows a prominent peak at $E_{x}=1.46 \mathrm{MeV}$, which was interpreted as a narrow resonance of ${ }^{19} \mathrm{C}$ with $E_{\text {rel }}=0.9 \mathrm{MeV}$. The angular distribution corresponding to this peak was compared with a microscopic distorted wave Born Approximation (DWBA) analysis, leading to spin-parity assignment of $5 / 2^{+}$, assuming a $1 / 2^{+}$spin for the ground state.

The ${ }^{19} \mathrm{C}$ is a one-neutron halo nucleus with a neutron separation energy of $S_{n}=0.589 \mathrm{MeV}$ [2]. As in the case of other one-neutron halo nuclei, such as ${ }^{11} \mathrm{Be}$, it is appealing to describe the scattering of ${ }^{19} \mathrm{C}$ by a stable target assuming that the projectile can be represented in terms of a simple core + valence neutron model, in which the ground state and the low-lying excited (possibly unbound) states are treated as pure single-particle states outside of a frozen core. On the other hand, theoretical calculations as well as knockout experiments [1,3-5] suggest important admixtures in the ground and excited states resulting from core-excited components. In this context, one of the purposes of this work is to investigate the adequacy of the ${ }^{18} \mathrm{C}+$ valence neutron model, where the core is assumed to be inert, to describe the resonant breakup data of Satou et al. [1].

Besides an adequate choice of the structure model, a meaningful interpretation of this reaction requires a realistic description of the reaction mechanism and the potentials involved in the calculations. In a recent analysis of the ${ }^{11} \mathrm{Be}$ $+p$ breakup at $63.7 \mathrm{MeV} /$ nucleon [6], based on a three-body model $\left({ }^{10} \mathrm{Be}+n+p\right)$, we found that an accurate representation of the $p-n$ interaction was crucial, while different choices of the underlying proton-core potential produced changes smaller than $10 \%$. Therefore, another motivation of the present work is to test the sensitivity of the calculated observables to the underlying neutron-proton interaction. Finally, to test the description of the reaction mechanism, we compare two different few-body approaches, namely, the Faddeev-Alt, Grassberger, Sandhas (Faddeev AGS) [7-9] and the continuum-discretized coupled-channels (CDCC) [10] methods. The CDCC method has a long history of success regarding the interpretation of reactions involving the scattering of two-body weakly bound projectiles on light and heavy targets [11-13] and, more recently, the scattering of three-body weakly bound projectiles [14-16]. On the other hand, the Faddeev AGS formalism has proved to be a very promising reaction tool to assess the validity of the approximations used in traditional scattering frameworks [17-20].

In summary, in this work we analyze the resonant breakup of ${ }^{19} \mathrm{C}$ on a proton target in order assess the validity of the singleparticle excitation mechanism within an inert core + valence neutron model, the sensitivity on the dynamical inputs (in particular the valence-target interaction), and the description of the reaction mechanism.

\section{THE OBSERVABLES}

We consider the breakup of a radioactive beam involving a two-body halo nucleus assumed to be well described by a core $(C)$ and a valence neutron $(n)$; the halo nucleus collides with a proton $(p)$ target leading to three free particles in the final state $(p, n, C)$. 
This final state is described in terms of nine kinematical variables. Momentum and energy conservation reduces this number to five independent variables. The kinematic configurations of the three-body breakup can be characterized by the polar and azimuthal angles $\Omega_{i}=\left(\theta_{i}, \phi_{i}\right)$ of the detected particles, as well as their respective momenta.

In many actual experiments, such as the one we are analyzing in the present work, the measured data is expressed in the relative (rel) core-valence energy $E_{\text {rel }}$, and the angles $d \Omega_{\text {c.m. }}$. between the $C-n$ final momentum and the beam direction in the center of mass (c.m.) system. Therefore, one needs to evaluate the double differential cross section $d^{2} \sigma / d E_{\text {rel }} d \Omega_{\text {c.m. }}$. To obtain the angular distribution corresponding to a given resonance, $d \sigma / d \Omega_{\mathrm{c} . \mathrm{m}}$, one needs to integrate over the resonance region leading to the single differential cross section.

We discuss in detail how these reaction observables are calculated in each reaction framework.

\section{THE REACTION APPROACH}

The study of the scattering of a projectile nucleus from a structureless proton target is the simplest reaction where one only needs to identify the dominant degrees of freedom of the projectile.

The treatment of the projectile can range from a pure microscopic approach, where the projectile excitation is treated in terms of single-particle excitations of all active nucleons, to a collective approach, where excitations are described by the shape deformation of the nucleus as a whole. In the case of a one-neutron halo projectile, one may assume that a good description for such nucleus is a cluster model based on an inert core plus a valence neutron. If the core has low-lying excited states, these can be excited during the collision process.

Three-body reaction formalisms such as the Faddeev AGS and the CDCC have been developed and used to study the scattering of halo nuclei. An appealing characteristic of these reaction frameworks is the simultaneous treatment of the resonant and nonresonant channels. In the case of the CDCC method, recent developments include the effect of core excitation [21]. This feature is still absent in existing implementations of the full multiple scattering Faddeev AGS formalism. First steps were made to include core excitation in the first-order term of the multiple scattering expansion of the Faddeev AGS approach [22].

For completeness, we summarize in Subsecs. A and B the inert core Faddeev AGS and CDCC reaction frameworks, respectively.

\section{A. The Faddeev AGS formalism}

The Faddeev AGS approach is a three-body scattering formalism that treats all three-pair partitions on equal footing. All open channels (elastic, inelastic, transfer, and breakup) are calculated simultaneously from first principles. In this framework, if one considers the scattering from an initial state $\alpha$ to a final state $\beta$ with $\alpha, \beta=(1,2,3)$ in the odd-man-out notation, the three-body AGS operators $U^{\beta \alpha}[8]$ (which are equivalent to the usual transition amplitudes on the energy shell) are obtained from the solution of an integral equation (see Refs. [9,19] for a derivation) that is written as

$$
U^{\beta \alpha}=\bar{\delta}_{\beta \alpha} G_{0}^{-1}+\sum_{\gamma} \bar{\delta}_{\beta \gamma} t_{\gamma} G_{0} U^{\gamma \alpha},
$$

where $\bar{\delta}_{\beta \alpha}=1-\delta_{\beta \alpha}$. The two-body transition operator for each interacting pair is

$$
t_{\gamma}=v_{\gamma}+v_{\gamma} G_{0} t_{\gamma}
$$

where $v_{\gamma}$ is the interaction between the pair $\gamma$ and $G_{0}$ is the free resolvent

$$
G_{0}=\left(E+i 0-H_{0}\right)^{-1} .
$$

In here, $E$ is the total energy of the three-particle system in the center of mass (c.m.) and $H_{0}$ is the kinetic energy operator for the three particles.

The scattering amplitudes are the matrix elements of $U^{\beta \alpha}$ calculated between initial and final states that are eigenstates of the corresponding channel Hamiltonian $H_{\alpha}\left(H_{\beta}\right)=H_{0}+v_{\alpha}\left(v_{\beta}\right)$ with the same energy eigenvalue. For breakup, the final state is the product of two plane waves corresponding to the relative motion of three free particles that may be expressed in any of the relative Jacobi variables.

The numerical solution of the AGS equations can be found by summing the corresponding Neumann series using the Padé method [23]. In our calculations, Eq. (1) is solved in momentum space after partial wave decomposition and discretization of all momentum variables. We include the nuclear interaction between all three pairs and the Coulomb interaction between the proton and ${ }^{18} \mathrm{C}$, following the technical developments implemented in Refs. [24] and [25].

Since in the c.m. one has $\Omega_{p}=-\Omega_{\text {c.m. }}$, for the purpose of analyzing the data, one chooses the Jacobi momenta for the final breakup state,

$$
\begin{aligned}
& \mathbf{p}=\frac{m_{n} \mathbf{K}_{C}-m_{C} \mathbf{K}_{n}}{m_{C}+m_{n}}, \\
& \mathbf{q}=\frac{\left(m_{C}+m_{n}\right) \mathbf{K}_{p}-m_{p}\left(\mathbf{K}_{C}+\mathbf{K}_{n}\right)}{M},
\end{aligned}
$$

with $\mathbf{K}_{p}, \mathbf{K}_{n}, \mathbf{K}_{C}\left(m_{p}, m_{n}, m_{C}\right)$ being the laboratory momenta (masses) of the proton, valence neutron, and core in the exit system, respectively, and $M=m_{p}+m_{n}+m_{C}$. The breakup differential cross section is calculated from the on-shell matrix element of the AGS operators $T^{0 \alpha}=\left\langle\mathbf{q p}\left|U^{0 \alpha}\right| \psi_{\alpha}\right\rangle$, where particle $\alpha$ is the spectator in the initial state (in our case, $\alpha$ is the proton) and $\psi_{\alpha}$ is an eigenstate of $H_{\alpha}$. The Jacobi momenta in the final-state plane wave satisfy the on-shell relation

$$
E-\frac{p^{2}}{2 \mu}-\frac{q^{2}}{2 \bar{\mu}}=0
$$

with the reduced masses

$$
\begin{aligned}
\mu & =\frac{m_{n} m_{C}}{m_{n}+m_{C}}, \\
\bar{\mu} & =\frac{m_{p}\left(m_{n}+m_{C}\right)}{M} .
\end{aligned}
$$


The semi-inclusive cross section for the measured proton is, following an identical derivation as in Ref. [17],

$$
\begin{aligned}
& \frac{d^{2} \sigma}{d \Omega_{p} d E_{p}} \\
& =(2 \pi)^{4} \frac{m_{n}+m_{C}}{K_{\mathrm{LAB}}} m_{p} \mathcal{K}_{p} \int d^{2} \hat{p}\left|T^{0 \alpha}\right|^{2} \\
& \quad \times \mu \sqrt{2 \mu E-\frac{\mu}{\bar{\mu}}\left(\mathcal{K}_{p}^{2}+\frac{m_{p}^{2}}{M^{2}} \mathcal{K}_{\text {TOT }}^{2}-2 \frac{m_{p}}{M} \mathcal{K}_{p} \cdot \mathcal{K}_{\mathrm{TOT}}\right),}
\end{aligned}
$$

where $\mathcal{K}_{\mathrm{TOT}}=\mathcal{K}_{n}+\mathcal{K}_{p}+\mathcal{K}_{C}$ may be the total momentum in the laboratory where $\mathcal{K}_{\mathrm{TOT}}=K_{\mathrm{LAB}}$, or in the c.m. $\mathcal{K}_{\mathrm{TOT}}=0$. Then, in order to obtain the dependence of the calculated breakup observable in terms or the relative coreneutron energy, we use the relation

$$
E_{\text {rel }}=E-\left(1+\frac{m_{p}}{m_{C}+m_{p}}\right) E_{p},
$$

which is valid in the c.m. frame where in addition $\theta_{\text {c.m. }}=\pi-\theta_{p}$. In the Faddeev-AGS approach it is not possible to disentangle the contribution to the cross section from each breakup state. Labeling the three interacting particles $(p C n)$ as (123), the AGS breakup operator $U^{01}$ is obtained by quadrature,

$$
U^{01}=t_{1} G_{0} U^{11}+t_{2} G_{0} U^{21}+t_{3} G_{0} U^{31} .
$$

For the case of resonant breakup, the term $t_{1} G_{0} U^{11}$ is dominant, but nonresonant breakup equally contributes to each term of the sum. Thus, we cannot single out the resonant contribution in the same way as done in other scattering frameworks such as CDCC. However, we can extract the resonant contribution in the following way. First, we calculate the AGS operators without the resonant term. This can be done either by taking away the dominant term $t_{1} G_{0} U^{11}$ or by not including the $n{ }^{18} \mathrm{C}$ interaction in the $d$ wave. The resonant contribution is then evaluated by subtracting the breakup cross section without the resonant term from the full breakup cross section.

\section{B. The CDCC formalism}

The CDCC framework was developed to study three-body nuclear reactions in which a weakly bound two-body projectile scatters from a target nucleus. This formalism has been extensively applied to reactions induced by halo nuclei with one nucleon (neutron or proton) outside the core, and standard CDCC applications also assume (as standard Faddeev AGS) a single core state.

This formalism consists in solving the Schrödinger equation in a model space in which the three-body wave function is expanded in the internal states (bound and continuum states) of the two-body projectile. In this way, CDCC can treat elastic, inelastic, and breakup channels simultaneously but cannot include transfer, since it does not treat all three-body partitions in equal footing.

The projectile internal Hamiltonian $H_{p}$ has a finite number of bound states and an infinite number of continuum energy states. In order to study the breakup of a weakly bound projectile, we need to include these scattering states. For practical reasons, CDCC makes use of the bin method to discretize the continuum. In this method, the energy spectrum is truncated at a maximum excitation energy $\varepsilon_{\max }$ and divided into intervals. For each energy interval, called bin, a representative square-integrable state is constructed as a linear superposition of the two-body scattering states in that interval. Thus, the radial functions are defined as follows:

$$
\bar{u}_{\alpha}(r)=\sqrt{\frac{2}{\pi N_{\alpha}}} \int_{k_{\alpha-1}}^{k_{\alpha}} f_{\alpha}(k) u_{\alpha}(k, r) d k,
$$

where $u_{\alpha}(k, r)$ are the continuum radial wave functions with wave number $k=\sqrt{2 m \varepsilon} / \hbar$ and $\alpha$ is an appropriate set of quantum numbers. Here $f_{\alpha}(k)$ is a weight function and $N_{\alpha}$ is its normalization constant. The bound and bin states are taken as the states of the projectile pair and denoted as $\phi_{\alpha}(\mathbf{r})$.

Let $\mathbf{K}_{0}$ be the incident wave number of the projectile in the c.m. frame. The exact three-body wave function $\Psi_{\mathbf{K}_{0}}^{+}(\mathbf{R}, \mathbf{r})$ satisfies the Schrödinger equation

$$
(H-E) \Psi_{\mathbf{K}_{0}}^{+}(\mathbf{R}, \mathbf{r})=0,
$$

where $H$ is the three-body Hamiltonian, $E$ is the total c.m. energy of the system, $\mathbf{R}$ is the relative distance between the c.m. of the projectile and the target, and $\mathbf{r}$ is the relative distance between the valence particle and the core.

The Hamiltonian $H$ for this system can be written as a sum of three terms,

$$
H=T_{R}+U_{p t}(\mathbf{R}, \mathbf{r})+H_{p},
$$

where $T_{R}$ is the c.m. kinetic energy, $U_{p t}$ is the potential between the projectile constituents and the target

$$
U_{p t}(\mathbf{R}, \mathbf{r})=\sum_{j=C, v} V_{j t}(\mathbf{R}, \mathbf{r}),
$$

and $H_{p}$ is the internal Hamiltonian of the projectile. In the CDCC approach, the exact wave function is expressed as an expansion in terms of the states (bound and continuum bins) $\phi_{\alpha}(\mathbf{r})$ of the two-body Hamiltonian $H_{p}$ as follows:

$$
\Psi_{\mathbf{K}_{0}}^{\mathrm{CDCC}}(\mathbf{R}, \mathbf{r})=\sum_{\alpha=0}^{N} \phi_{\alpha}(\mathbf{r}) \omega_{\alpha}(\mathbf{R}),
$$

where $\alpha=0$ refers to the projectile ground state. The bin states includes both the resonant and the nonresonant part of the continuum. The wave functions $\omega_{\alpha}(\mathbf{R})$ of the projectile-target relative motion are solutions of the coupled-channels equations

$$
\left(E_{\alpha}-T_{R}-V_{\alpha \alpha}(\mathbf{R})\right) \omega_{\alpha}(\mathbf{R})=\sum_{\beta \neq \alpha} V_{\alpha \beta}(\mathbf{R}) \omega_{\beta}(\mathbf{R}),
$$

where $E_{\alpha}=E-\varepsilon_{\alpha}$ and the coupling potentials are

$$
V_{\alpha \beta}(\mathbf{R})=\left\langle\phi_{\alpha}\left|U_{p t}(\mathbf{R}, \mathbf{r})\right| \phi_{\beta}\right\rangle .
$$

From the solution of the coupled equations (15), one obtains the radial functions $\omega_{\alpha}$, whose asymptotic form provides the $S$-matrix elements $S_{0 \alpha}$ for the transition from the initial-state to the final-state $\alpha$. 
Energy conservation relates the wave numbers $K_{\alpha}$ with the state energies $\varepsilon_{\alpha}$ as

$$
\frac{\hbar^{2} K_{\alpha}^{2}}{2 \mu_{p t}}+\varepsilon_{\alpha}=\frac{\hbar^{2} K_{0}^{2}}{2 \mu_{p t}}+\varepsilon_{0} .
$$

The inelastic differential cross-section angular distribution, in c.m. frame, for excitation of a given state is

$$
\frac{d \sigma_{\alpha}}{d \Omega_{\mathrm{c} . \mathrm{m} .}}=\left(\frac{\mu_{p t}}{2 \pi \hbar^{2}}\right)^{2} \frac{K_{\alpha}}{K_{0}}\left|T^{\alpha}\left(\mathbf{K}_{\alpha}\right)\right|^{2},
$$

with $T^{\alpha}\left(\mathbf{K}_{\alpha}\right)$ denoting the transition amplitude leading to the state $\alpha$. Then, we can obtain the triple differential cross section for the excitation of any bin state $\alpha$ (resonant or nonresonant) with respect to the solid angle and the relative energy, dividing by the energy width of the bin (either resonant or nonresonant) as

$$
\frac{d^{2} \sigma}{d \Omega_{\mathrm{c} . \mathrm{m} .} d \varepsilon_{\alpha}} \approx \frac{1}{\Delta \varepsilon_{\alpha}}\left(\frac{d \sigma_{\alpha}}{d \Omega_{\mathrm{c} . \mathrm{m} .}}\right),
$$

where $\Delta \varepsilon_{\alpha}$ denotes the width of the continuum bin $\alpha$.

\section{THE PAIR INTERACTIONS}

In both the Faddeev AGS and CDCC approaches, one needs to define the three-pair interactions that in our case study are the $p-n, p-{ }^{18} \mathrm{C}$, and $n-{ }^{18} \mathrm{C}$ interactions.

For the purpose of comparing the CDCC and Faddeev AGS scattering frameworks we take for $p$ - $n$ the Gaussian potential of Ref. [10], with parameters adjusted to reproduce the deuteron rms radius and binding energy, as well as the triplet ${ }^{3} S$ phase shifts. We also use the realistic nucleon-nucleon CD-Bonn potential [26]. For the potential between the proton and ${ }^{18} \mathrm{C}$ core, we use a phenomenological optical model with parameters taken from the global parametrization of Watson et al. [19,27], evaluated at the appropriate energy per nucleon.

For the $n^{-18} \mathrm{C}$ interaction, we adopt a simple central potential

$$
V(r)=-V_{c} f\left(r, R_{c}, a_{c}\right),
$$

where $f(r, R, a)$ is the usual Woods-Saxon form factor,

$$
f\left(r, R_{c}, a\right)=1 /\left\{1+\exp \left[\left(r-R_{c}\right) / a\right]\right\},
$$

with $R_{c}=r_{0} A^{\frac{1}{3}}$. We use the geometry $r_{0}=1.2 \mathrm{fm}$ and $a_{0}=0.6 \mathrm{fm}$. To reproduce the separation energy of the ground state and the position of the $5 / 2^{+}$resonance, the depth is assumed to be $\ell$ dependent. The $s$-wave interaction is adjusted to generate a $2 s$ bound state with energy $E(2 s)=-0.6 \mathrm{MeV}$. This interaction also supports a Pauli forbidden $1 s$ bound state with energy $E(1 s)=-22.1 \mathrm{MeV}$. In the Faddeev AGS calculations, this state is projected out by moving it to large positive energy. In the CDCC method, this state is just omitted in the calculations. The interaction in any other waves, other than the $d$ wave, is chosen to be the same as in the $s$ wave. The interaction in the $p$ wave generates a $1 p$ bound state with energy $E(1 p)=-9.6 \mathrm{MeV}$, which is also projected out by moving it to large positive energy. The interaction in the $d$ wave is adjusted to generate a $1 d$ resonance with energy $E(1 d)=0.9-0.06 \mathrm{i} \mathrm{MeV}$ with a very narrow width.

\section{RESULTS}

We present now the results for the angular distribution of the resonant breakup $p+{ }^{19} \mathrm{C}\left(1 / 2^{+}\right) \rightarrow p+{ }^{19} \mathrm{C}\left(5 / 2^{+}\right)$due to the single-particle excitation of the valence neutron, with the core kept in the $0_{1}^{+}$ground state.

In the solution of the Faddeev AGS equations, we include $n-p$ partial waves up to relative orbital angular momentum $L \leqslant 4, p-{ }^{18} \mathrm{C}$ up to $L \leqslant 15$, and $n-{ }^{18} \mathrm{C}$ up to $L \leqslant 4$. Three-body total angular momentum is included up to $J=100$. In the CDCC calculation, we include up to $L=70$ in the projectiletarget relative angular momentum and the coupled equations are integrated up to a matching radius $R_{\text {match }}=80 \mathrm{fm}$. For the ${ }^{19} \mathrm{C}$ states, we considered $\ell=0-3$ partial waves. For each $\ell$, the ${ }^{19} \mathrm{C}$ continuum was truncated at a maximum excitation energy of $40 \mathrm{MeV}$ and discretized into 70 bins (in order to get an accurate energy distribution).

In Fig. 1, we show the differential breakup cross-section energy distribution. The distribution presents a pronounced peak around the energy of the single-particle resonance. As shown in the figure, the calculated observables using both reaction formalisms (the Faddeev AGS and the CDCC) and using the same $L$-independent Gaussian NN interaction are very similar at low relative energies and around the resonance. However, the calculations start to differ at large excitation energies.

In Fig. 2, we compare the calculated differential breakup cross-section angular distribution with the data taken from Ref. [1]. For a meaningful comparison, the calculations are integrated in the energy region of the resonance, that is, including the peak of Fig. 1.

The angular distribution calculated with the Faddeev AGS approach, using the Gaussian potential (solid line), is very similar to the corresponding predictions of the CDCC approach (dashed line). Both calculations have the same order of magnitude of the experimental data; however, the shape is rather different. Upon substituting the Gaussian potential by the realistic CD-Bonn NN potential (dash-dotted curve) in the

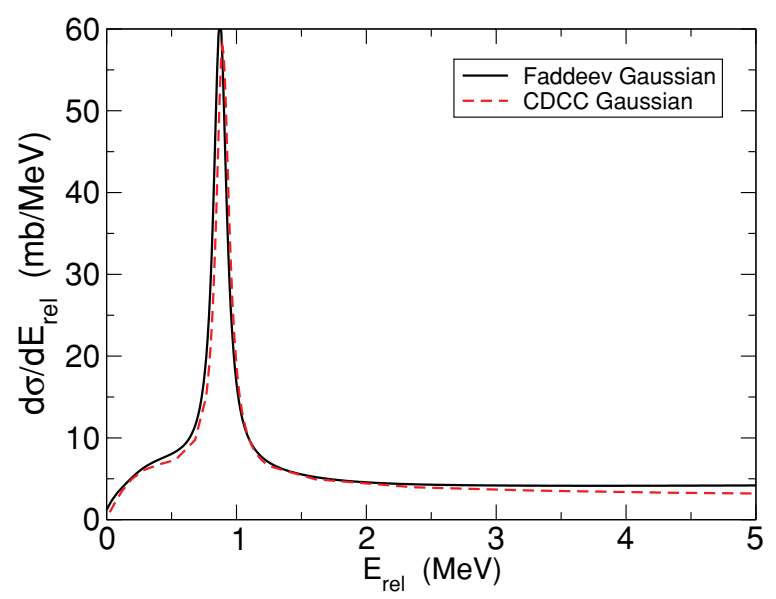

FIG. 1. (Color online) Calculated differential energy distribution for the resonant breakup $p\left({ }^{19} \mathrm{C}, p\right){ }^{18} \mathrm{C} n$ at $70 \mathrm{MeV} /$ nucleon. The solid curve corresponds to Faddeev AGS reaction framework and the dashed line is the result of the CDCC calculation, used in both the Gaussian NN interactions. 


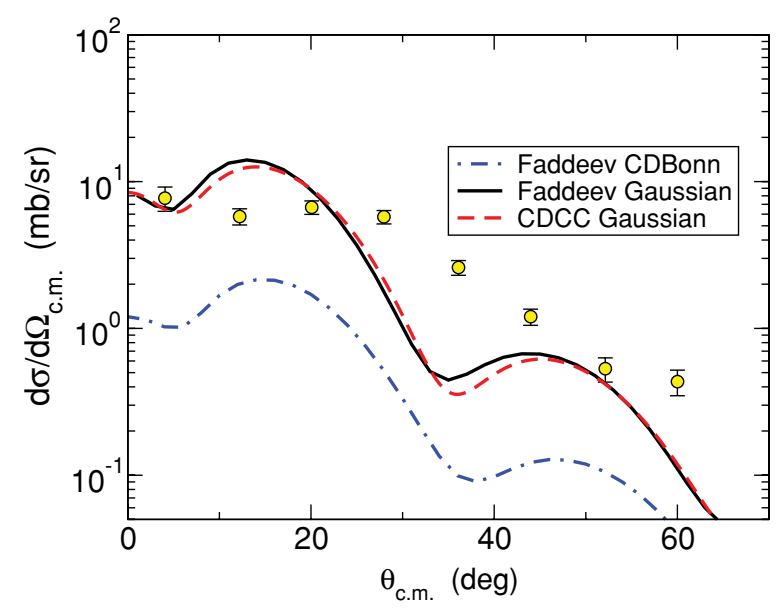

FIG. 2. (Color online) Experimental and calculated differential angular distribution for the resonant breakup $p\left({ }^{19} \mathrm{C}, p\right){ }^{18} \mathrm{C} n$ at $70 \mathrm{MeV} /$ nucleon. The solid (dash-dotted) curve corresponds to Faddeev AGS reaction framework using the Gaussian (CD-Bonn) $\mathrm{NN}$ interaction. The dashed line is the result of the CDCC calculation using the Gaussian interaction.

Faddeev AGS formalism, the result is drastically decreased by an order of magnitude. Thus, the angular distribution for the resonant breakup is very sensitive to a realistic treatment of the $\mathrm{NN}$ potential, an effect even more significant than that found in the work of Ref. [6].

No calculation is able to reproduce the shape of the angular distribution independently of the $n-p$ potential that is used. We note that we have assumed pure single-particle configurations for the ground and resonant states. This single-particle transition is expected to be even smaller when the renormalization due to the spectroscopic factor is introduced. We have also evaluated other single-particle transitions where the core is kept in the same state. We found that the transition where the core remains in its excited $2_{1}^{+}$state and the valence nucleon makes a transition from the $1 d_{5 / 2}$ to the $2 s_{1 / 2}$ configuration is two orders of magnitude smaller than the data and thus also negligible.

Therefore, we can conclude that single-particle transitions where the core remains in the same state may be neglected because they underpredict the data by an order of magnitude. Consequently, we expect that, within a core + valence picture, the main contribution to the resonant breakup should arise from the core-excitation contributions. These effects have been investigated at length in early studies of inelastic excitation of stable nuclei within the microscopic DWBA approach. These studies identified situations in which the inclusion of these core polarization effects are essential to account for the experimental cross section. Within a microscopic picture, this result was interpreted as a consequence of the limited number of shell-model configurations included in the microscopic calculation. In this context, the core polarization can be taken into account by enlarging the model space used in the microscopic inelastic form factors or by using an effective NN interaction [28]. Within a core + valence two-body approach, as used here, this effect can be included, incorporating explicitly the excitation of the core within a macroscopic (collective) picture. This idea has been used in the past to analyze the inelastic scattering of stable nuclei, such as ${ }^{90} \mathrm{Zr}$ [29]. A similar idea has been applied in an extended version of the CDCC formalism [21]. More recently, the effect of core excitation was also used within a simple reaction mechanism to explain the breakup of ${ }^{11} \mathrm{Be}$ on a proton target, containing integrated contributions with excitation energies in the range $E_{x}=3.0-5.5 \mathrm{MeV}$ [22].

To get a more quantitative idea of the relevance of coreexcited components in the states involved in this analysis, we have performed shell-model calculations for the ${ }^{19} \mathrm{C}$ nucleus, using the code OXBASH [30] with the spsdpf model space and the Warburton-Brown (WBP) interaction [31]. These calculations predict a $1 / 2^{+}$ground state for ${ }^{19} \mathrm{C}$ with a dominant ${ }^{18} \mathrm{C}\left(0^{+}\right) \otimes 2 s_{1 / 2}$ configuration with spectroscopic factor $S=0.58$, but with non-negligible contributions of core-excited components. Similarly, the resonance reported by Satou et al. [1] at $1.46 \mathrm{MeV}$ is likely to correspond to the $5 / 2_{2}^{+}$shell-model state calculated at $E_{x}=1.56 \mathrm{MeV}$. For this state, the shell-model calculation predicts the dominant configurations ${ }^{18} \mathrm{C}\left(2_{1}^{+}\right) \otimes 2 s_{1 / 2}$ and ${ }^{18} \mathrm{C}\left(2_{1}^{+}\right) \otimes 1 d_{5 / 2}$ with spectroscopic factors 0.61 and 0.29 , respectively. Consequently, the core-excitation mechanism is expected to play a significant role in the transition between these two states.

Since our results show that within the core + valence neutron model the single-particle mechanism gives a negligible contribution to the calculated resonant breakup, we expect that the core-excitation mechanisms should be the dominant reaction mechanism as suggested by the number of shell-model configurations involved in the initial and final ${ }^{19} \mathrm{C}$ states.

\section{SUMMARY AND CONCLUSIONS}

We have performed full Faddeev-type and CDCC calculations for proton inelastic scattering from ${ }^{19} \mathrm{C}$ at $70 \mathrm{MeV} /$ nucleon incident energies, using a two-body model for ${ }^{19} \mathrm{C}$ consisting of an inert ${ }^{18} \mathrm{C}$ core and a valence neutron.

We have shown that the Faddeev AGS results using a simple $L$-independent $p$ - $n$ potential of Gaussian parametrization agree fairly well with the corresponding CDCC calculations, using the same interaction. However, the calculated observable considerably differs from the one obtained with a realistic CD-Bonn potential. The former is found to overestimate the nonresonant breakup angular distributions calculated with the realistic NN interaction by almost an order of magnitude. These results strongly suggest that, at least in this energy regime, the resonant breakup observable is very sensitive to the underlying $\mathrm{NN}$ interaction and that any reliable fewbody scattering framework should incorporate a realistic NN interaction.

We also conclude that the single-particle excitations of the valence particle where the core remains in the same state do not contribute significantly to the resonant breakup. Reaction calculations including core-excitation mechanism are in progress and will be presented elsewhere. The role of core excitation could shed light on the deformation of ${ }^{19} \mathrm{C}$ core, in particular if it has a prolate or oblate intrinsic deformation $[32,33]$. 


\section{ACKNOWLEDGMENTS}

We are grateful to Y. Satou for providing us the experimental data in tabular form. The authors are supported by the FCT
Grants PTDC/FIS/65736/2006 and PTDC/FIS/103902/2008, the Complementary Action Portugal-Spain PORT2008-05, and the Spanish Ministerio de Ciencia e Innovación under Project FPA2009-07653.
[1] Y. Satou et al., Phys. Lett. B 660, 320 (2008).

[2] G. Audi, A. H. Wapstra, and C. Thibault, Nucl. Phys. A 729, 337 (2003).

[3] V. Maddalena et al., Phys. Rev. C 63, 024613 (2001).

[4] S. Karataglidis, K. Amos, P. Fraser, L. Canton, and J. P. Svenne, Nucl. Phys. A 813, 235 (2008).

[5] P. Descouvement, Nucl. Phys. A 675, 559 (2000).

[6] E. Cravo, R. Crespo, A. M. Moro, and A. Deltuva, Phys. Rev. C 81, 031601 (2010).

[7] L. D. Faddeev, Zh. Eksp. Teor. Fiz. 39, 1459 (1960) [Sov. Phys. JETP 12, 1014 (1961)].

[8] E. O. Alt, P. Grassberger, and W. Sandhas, Nucl. Phys. B 2, 167 (1967).

[9] W. Glöckle, The Quantum Mechanical Few-Body Problem (Springer-Verlag, Berlin, 1983).

[10] N. Austern, Y. Iseri, M. Kamimura, M. Kawai, G. Rawitsher, and M. Yahiro, Phys. Rep. 154, 125 (1987).

[11] M. Yahiro, Y. Iseri, H. Kameyama, M. Kamimura, and M. Kawai, Prog. Theor. Phys. Suppl. 89, 32 (1986).

[12] F. M. Nunes and I. J. Thompson, Phys. Rev. C 59, 2652 (1999).

[13] J. A. Tostevin, F. M. Nunes, and I. J. Thompson, Phys. Rev. C 63, 024617 (2001).

[14] T. Matsumoto, T. Egami, K. Ogata, Y. Iseri, M. Kamimura, and M. Yahiro, Phys. Rev. C 73, 051602 (2006).

[15] M. Rodríguez-Gallardo, J. M. Arias, J. Gómez-Camacho, R. C. Johnson, A. M. Moro, I. J. Thompson, and J. A. Tostevin, Phys. Rev. C 77, 064609 (2008).

[16] M. Rodríguez-Gallardo, J. M. Arias, J. Gómez-Camacho, A. M. Moro, I. J. Thompson, and J. A. Tostevin, Phys. Rev. C 80, 051601(R) (2009).
[17] R. Crespo, A. Deltuva, M. Rodríguez-Gallardo, E. Cravo, and A. C. Fonseca, Phys. Rev. C 79, 014609 (2009).

[18] R. Crespo, A. Deltuva, E. Cravo, M. Rodríguez-Gallardo, and A. C. Fonseca, Phys. Rev. C 77, 024601 (2008).

[19] R. Crespo, E. Cravo, A. Deltuva, M. Rodríguez-Gallardo, and A. C. Fonseca, Phys. Rev. C 76, 014620 (2007).

[20] A. Deltuva, A. M. Moro, E. Cravo, F. M. Nunes, and A. C. Fonseca, Phys. Rev. C 76, 064602 (2007).

[21] N. C. Summers, F. M. Nunes, and I. J. Thompson, Phys. Rev. C 74, 014606 (2006).

[22] R. Crespo, A. Deltuva, and A. M. Moro, Phys. Rev. C 83, 044622 (2011).

[23] G. A. Baker and J. L. Gammel, eds., The Padé Approximation in Theoretical Physics (Academic, New York, 1970).

[24] A. Deltuva, A. C. Fonseca, and P. U. Sauer, Phys. Rev. C 71, 054005 (2005); 72, 054004 (2005); 73, 057001 (2006).

[25] A. Deltuva, Phys. Rev. C 74, 064001 (2006).

[26] R. Machleidt, Phys. Rev. C 63, 024001 (2001).

[27] B. A. Watson, P. P. Singh, and R. E. Segel, Phys. Rev. 182, 978 (1969).

[28] W. G. Love and G. R. Satchler, Nucl. Phys. A 101, 424 (1967).

[29] A. M. Kobos, B. A. Brown, R. Lindsay, and G. R. Satchler, Nucl. Phys. A 425, 205 (1984).

[30] J. A. Brown, A. Etchegoyen, and W. D. M. Rae, MSU-NSCL Report No. 524, 1988 (unpublished).

[31] E. K. Warburton and B. A. Brown, Phys. Rev. C 46, 923 (1992).

[32] T. Suzuki et al., in Proceedings of the International Symposium on Frontiers of Collective Motions, edited by $\mathrm{H}$. Sagawa and H. Iwasaki (World Scientific, Singapore, 2003), p. 236

[33] H. Sagawa, X. R. Zhou, X. Z. Zhang, and T. Suzuki, Phys. Rev. C 70, 054316 (2004). 